\title{
On Limit Theorems for the Bivariate (Magnetization, Energy) Variable at the Critical Point
}

\author{
Joël De Coninck
}

Université de l'Etat, Faculté des Sciences, B-7000 Mons, Belgium

\begin{abstract}
The limiting (magnetization, energy) bivariate variable is studied for Ising ferromagnets at the critical point. The factorization property of the limiting bivariate moment generating function is shown to be intimately connected to critical point exponent inequalities and to the behaviour of the scaling limit near and at the critical point. The validity of this can be deduced from the study of the second and the fourth magnetization cumulants at zero external field. The limiting bivariate variable is exactly calculated at the critical point for the CurieWeiss model (MF) and for the edge of a two-dimensional Ising ferromagnet wrapped on a cylinder. It is shown that the mean field case leads to a nonGaussian limiting distribution in contradistinction with the particular Ising model we consider for which we obtain a product of two Gaussian probability distributions.
\end{abstract}

\section{Introduction}

Many recent investigations have been devoted to limit theorems for sums of dependent random variables occurring in classical spin models such as the magnetization and the ene:gy variables $[1-12]$. The main difficulty encountered in this field has to be found in the non-independent character of the elementary spin variables which describe the models.

Let us indeed consider for definiteness a classical Ising ferromagnet on $\mathbb{Z}^{d}$ defined by the usual Hamiltonian

$$
H\left(\left\{\sigma_{\Lambda}\right\}\right)=-\frac{1}{2} \sum_{i, j \in \Lambda} J_{i j} \sigma_{i} \sigma_{j}-h \sum_{i \in \Lambda} \sigma_{i},
$$

where $\Lambda \subset \mathbb{Z}^{d}, J_{i j} \geqq 0$ with $\sum_{j \in \mathbb{Z}^{d}} J_{i j}<+\infty,\left\{\sigma_{\Lambda}\right\}$ refers to a given configuration for $\Lambda$ and $h$ denotes the external field. The renormalized block magnetization variable $\tilde{M}_{\Lambda}$ and renormalized block energy variable $\widetilde{E}_{\Lambda}$ are given by

$$
\begin{aligned}
& M_{\Lambda}=\sum_{i \in \Lambda} \sigma_{i}, \\
& \tilde{M}_{\Lambda}=\left(M_{\Lambda}-\left\langle M_{\Lambda}\right\rangle\right) /|\Lambda|^{\left(\omega_{1 / 2},\right.},
\end{aligned}
$$


and

$$
\begin{aligned}
& E_{\Lambda}=-\frac{1}{2} \sum_{i, j \in \Lambda} J_{i j} \sigma_{i} \sigma_{j}, \\
& \tilde{E}_{\Lambda}=\left(E_{\Lambda}-\left\langle E_{\Lambda}\right\rangle\right) /|\Lambda|^{\omega_{2} / 2},
\end{aligned}
$$

where $\omega_{1}$ and $\omega_{2}$ have to be chosen such that the corresponding limit laws exist as $\Lambda \uparrow \mathbb{Z}^{d}$. The mean value $\langle\cdot\rangle$ has to be taken with respect to the probability distribution induced by (1), i.e.:

$$
P_{\beta, h}(\{\sigma\})=\exp \left(-\beta H\left(\left\{\sigma_{\Lambda}\right\}\right)\right) / \sum_{\left\{\sigma_{\hat{A}}\right.} \exp \left(-\beta H\left(\left\{\sigma_{\Lambda}\right\}\right)\right) .
$$

Within this context, we would like to focus our attention on the limiting behaviour of the bivariate moment generating function:

$$
\phi\left(t_{1}, t_{2}\right)=\lim _{\Lambda \uparrow \mathbb{Z}^{d}}\left\langle\exp \left(t_{1} \tilde{M}_{\Lambda}+t_{2} \tilde{E}_{\Lambda}\right)\right\rangle .
$$

Previous works have been devoted to the study of $\phi\left(t_{1}, 0\right)[1-11]$ or to $\phi\left(0, t_{2}\right)$ $[4,11,12]$. It is only recently that Newman achieved important progress along this line [13]: he has indeed proven that away from the possible two phase region in the $(\beta, h)$ - plane, the choice $\omega_{1}=\omega_{2}=1 / 2$ leads to (at least for bounded spins)

$$
\phi\left(t_{1}, t_{2}\right)=\exp \left(a t_{1}^{2}+b t_{2}^{2}+c t_{1} t_{2}\right),
$$

where $a, b$ and $c$ are real constants. This paper considerably generalizes previous results on the subject $[10,11]$. Let us also point out that at zero external field $(h=0)$, by symmetry (5) simply becomes

$$
\phi\left(t_{1}, t_{2}\right)=\exp \left(A t_{1}^{2}+B t_{2}^{2}\right)
$$

provided of course that $\beta<\beta_{c}$ which corresponds to the finiteness of the susceptibility. This result is quite remarkable since it implies that the reduced magnetization and energy variables become independent when $\Lambda \uparrow \mathbb{Z}^{d}$.

The situation at the critical point remains however an open question. The two main problems can be formulated as follows: (a) what are the possible limiting distributions associated to (4')? (b) do the reduced variables $\tilde{M}_{\Lambda}$ and $\widetilde{E}_{\Lambda}$ become independent when $\Lambda \uparrow \mathbb{Z}^{d}$ ?

The physical interest underlying this question may be found in the connection between the function $\phi\left(t_{1}, t_{2}\right)$ and the covariance of $\tilde{M}_{\Lambda}$ and $\tilde{E}_{\Lambda}$. It is indeed obvious that the nature of the limiting bivariate distribution depends on the correlation between the energy and the magnetization variables. According to the scaling theory of critical fluctuations, it is expected that, near the critical point and for large spin separation $r$, the spin-spin, energy density-energy density and energy density-spin correlation functions behave respectively as [21]

$$
\begin{aligned}
& f_{\sigma \sigma}(t, h, r)=L^{2(x-d)} f_{\sigma \sigma}(\bar{t}, \bar{h}, r / L), \\
& f_{e e}(t, h, r)=L^{2(y-d)} f_{e e}(\bar{t}, \bar{h}, r / L), \\
& f_{e \sigma}(t, h, r)=L^{x+y-2 d} f_{e \sigma}(\bar{t}, \bar{h}, r / L),
\end{aligned}
$$


with

$$
\begin{aligned}
L & =|\Lambda|^{1 / d}, \quad \bar{h}=h \cdot L^{x}, \\
t & =\left|\beta-\beta_{c}\right| / \beta_{c} \quad \text { and } \quad \bar{t}=t L^{y},
\end{aligned}
$$

where $x$ and $y$ are the usual scaling exponents of the correlation functions (7.a and $b$ ).

Integrating over $r$ implies that for large volumes $\Lambda$,

$$
\begin{aligned}
\left\langle M_{\Lambda} ; M_{\Lambda}\right\rangle & \sim|\Lambda|^{2 x / d}=|\Lambda|^{\omega_{1}}, \\
\left\langle E_{\Lambda} ; E_{\Lambda}\right\rangle & \sim|\Lambda|^{2 y / d}=|\Lambda|^{\omega_{2}}, \\
\left\langle M_{\Lambda} ; E_{\Lambda}\right\rangle & \sim|\Lambda|^{(x+y) / d},
\end{aligned}
$$

where, as usual,

$$
\langle A ; B\rangle=\langle A B\rangle-\langle A\rangle\langle B\rangle .
$$

The exponents $\omega_{1}$ and $\omega_{2}$ have been defined in (2) and (3). It may easily be proven that the exponent (if it exists) of the covariance of the magnetization and the energy variables is always less or equal to $\omega_{1}+\omega_{2}$. Whether or not this kind of inequalities reduces to an equality is precisely the cornerstone of the hyperscaling hypothesis and still remains an open question. In our probabilistic formulation of the problem, this property reveals itself in the nature of the limiting distribution: if $\widetilde{E}_{A}$ and $\widetilde{M}_{A}$ become independent when $\Lambda \uparrow \mathbb{Z}^{d}$, this means that there exists a strict inequality between the corresponding critical point exponents.

As will be shown later, this problem is also intimately connected with the behaviour of the scaling limit at the critical point and near the critical point (where the temperature of the block is a function of the size of the system $|\Lambda|$ and converges to the critical temperature as $|\Lambda| \rightarrow \infty)[12,23]$. To our knowledge at least, it is not known whether or not the scaling limit has to be unique: i.e. that the scaling limit at the critical point has to be identical to the scaling limit near the critical point. It will be shown in this paper that this unicity is connected with the independence of $\tilde{E}_{A}$ and $\tilde{M}_{\Lambda}$ as $\Lambda \uparrow \mathbb{Z}^{d}$.

The aim of this paper is therefore to give simple criteria to find out when $\tilde{M}_{\Lambda}$ and $\tilde{E}_{\Lambda}$ become independent as $\Lambda \uparrow \mathbb{Z}^{d}$. This will be developed in Sect. 2 . We also give two examples where the limiting bivariate moment generating function can exactly be computed at the critical point: the mean field where the limiting distribution is nonGaussian (Sect. 3) and the edge of a two-dimensional Ising ferromagnet where the limiting distribution is Gaussian and exhibits independence for the limiting variables (Sect. 4). Finally we present in Sect. 5 some concluding remarks and perspectives.

\section{General Considerations}

Let us for convenience introduce the function

$$
Z_{\Lambda}(x, y)=\sum_{\{\sigma\}} \exp \left\{\frac{x}{2} \sum_{i, j \in \Lambda} J_{i j} \sigma_{i} \sigma_{i}+y \sum_{i \in \Lambda} \sigma_{i}\right\} .
$$




\section{Defining}

$$
\Psi_{\Lambda}\left(t_{1}, t_{2}\right) \equiv\left\langle\exp \left(t_{1} M_{\Lambda}+t_{2} E_{\Lambda}\right)\right\rangle /\left\langle\exp \left(t_{1} M_{\Lambda}\right)\right\rangle\left\langle\exp \left(t_{2} E_{\Lambda}\right)\right\rangle,
$$

we easily obtain, at zero external field,

$$
\Psi_{\Lambda}\left(t_{1}, t_{2}\right)=\frac{\exp \left\{\log Z_{\Lambda}\left(\beta-t_{2}, t_{1}\right)-\log Z_{\Lambda}\left(\beta-t_{2}, 0\right)\right\}}{\exp \left\{\log Z_{\Lambda}\left(\beta, t_{1}\right)-\log Z_{\Lambda}(\beta, 0)\right\}}
$$

This may also be written in a more compact form as

$$
\Psi_{\Lambda}\left(t_{1}, t_{2}\right)=\left\langle\exp \left(t_{1} M_{\Lambda}\right)\right\rangle_{\beta-t_{2}, 0} /\left\langle\exp \left(t_{1} M_{\Lambda}\right)\right\rangle_{\beta, 0},
$$

where $\langle\cdot\rangle_{\beta, 0}$ denotes an expectation value with respect to (4) at temperature $\beta^{-1}$ and external field 0 . In terms of the reduced variables $\widetilde{M}_{\Lambda}$ and $\widetilde{E}_{\Lambda}$ introduced in (2) and (3), define

$$
\phi_{\Lambda}\left(t_{1}, t_{2}\right) \equiv\left\langle\exp \left(t_{1} \tilde{M}+t_{2} \tilde{E}_{\Lambda}\right)\right\rangle_{\beta, 0} /\left\langle\exp \left(t_{1} \tilde{M}_{\Lambda}\right)\right\rangle_{\beta, 0} \cdot\left\langle\exp \left(t_{2} \tilde{E}_{\Lambda}\right)\right\rangle_{\beta, 0},
$$

then

$$
\phi_{\Lambda}\left(t_{1}, t_{2}\right)=\left\langle\exp \left(t_{1} \tilde{M}_{\Lambda}\right)\right\rangle_{\beta-\tilde{t}_{2}, 0} /\left\langle\exp \left(t_{1} \tilde{M}_{\Lambda}\right)\right\rangle_{\beta, 0}
$$

with

$$
\tilde{t}_{2}=t_{2} /|\Lambda|^{\omega_{2} / 2}
$$

We are thus led to the

Theorem. 1. With the previously defined notations and for a fixed temperature $\beta^{-1}$, if the normalization exponents $\omega_{1}$ and $\omega_{2}$ are such that

$$
\begin{gathered}
\tilde{M}_{\Lambda} \rightarrow X, \\
\tilde{E}_{\Lambda} \rightarrow Y,
\end{gathered}
$$

as $|\Lambda| \rightarrow \infty$ in the sense of the weak convergence of probability distributions, then, for any $t_{1}, t_{2} \in \mathbb{R}$,

$$
E\left\{\exp \left(t_{1} X+t_{2} Y\right)\right\}=E\left\{\exp \left(t_{1} X\right)\right\} \cdot E\left\{\exp \left(t_{2} Y\right)\right\}
$$

if, and only if,

$$
\lim _{|\Lambda| \rightarrow \infty}\left\langle\exp \left(t_{1} \tilde{M}_{\Lambda}\right)\right\rangle_{\beta-\tilde{t}_{2}, 0}=E\left\{\exp \left(t_{1} X\right)\right\}
$$

or, in other words,

$$
\text { if, and only if, }
$$

the scaling limits near $\beta$ (according to $\beta-\tilde{t}_{2}$ ) and at $\beta$ are identical.

We now turn our attention to criteria for (20). The main difficulty lies of course in the fact that the exact determination of $\left\langle\exp \left(t_{1} \tilde{M}_{\Lambda}\right)\right\rangle_{\beta-\tilde{t}_{2}, 0}$ requires the knowledge of the partition function at non-zero external field. To avoid such a difficulty we shall derive useful inequalities at zero external field.

Using the Lee-Yang theorem [14」(which is satisfied for Ising ferromagnets), one may easily prove [15] that there exists a non-decreasing bounded function $K_{\Lambda}(x)$ 
such that

$$
\log Z_{\Lambda}(x, y)-\log Z_{\Lambda}(x, 0)=+\int_{-\infty}^{+\infty} \frac{1-\cos (y w)}{w^{2}} \mathrm{~d} K_{\Lambda}(w) .
$$

Let the integrated Ursell function of order $n$ be denoted by $U_{n}^{\Lambda}(x, y)$ :

$$
U_{n}^{\Lambda}(x, y)=\partial_{y}^{n} \log Z_{\Lambda}(x, y)
$$

One has $(n \geqq 1)$

$$
U_{n}^{\Lambda}(x, y)=\int_{\mathbb{R}} \partial_{y}^{n}(-\cos (y w)) \frac{1}{w^{2}} d K_{\Lambda}(w),
$$

and in particular

$$
\begin{gathered}
U_{2}^{\Lambda}(x, 0)=\int_{\mathbb{R}} d K_{\Lambda}(w), \\
U_{4}^{\Lambda}(x, 0)=-\int_{\mathbb{R}} w^{2} d K_{\Lambda}(w) .
\end{gathered}
$$

Since for any real $y$ :

$$
\frac{y^{2}}{2 !}-\frac{y^{4}}{4 !} \leqq 1-\cos (y) \leqq \frac{y^{2}}{2 !}
$$

one easily gets from $(21-24)$ that

$$
\exp \left\{\frac{t_{1}^{2}}{2 !} U_{2}^{\Lambda}(\beta, 0)+\frac{t_{1}^{4}}{4 !} U_{4}^{\Lambda}(\beta, 0)\right\} \leqq\left\langle\exp \left(t_{1} M_{\Lambda}\right)\right\rangle_{\beta, 0} \leqq \exp \left\{\frac{t_{1}^{2}}{2 !} U_{2}^{\Lambda}(\beta, 0)\right\} .
$$

One therefore has for $\phi_{\Lambda}\left(t_{1}, t_{2}\right)$ as introduced in (16):

$$
\begin{gathered}
\exp \left\{\frac{\tilde{t}_{1}^{2}}{2 !}\left[U_{2}^{\Lambda}\left(\beta-\tilde{t}_{2}, 0\right)-U_{2}^{\Lambda}(\beta, 0)\right]+\frac{\tilde{t}_{1}^{4}}{4 !} U_{4}^{\Lambda}\left(\beta-\tilde{t}_{2}, 0\right)\right\} \\
\leqq \Phi_{\Lambda}\left(t_{1}, t_{2}\right) \leqq \\
\exp \left\{\frac{\tilde{t}_{1}^{2}}{2 !}\left[U_{2}^{\Lambda}\left(\beta-\tilde{t}_{2}, 0\right)-U_{2}^{\Lambda}(\beta, 0)\right]-\frac{\tilde{t}_{1}^{4}}{4 !} U_{4}^{\Lambda}(\beta, 0)\right\}
\end{gathered}
$$

with

$$
\tilde{t}_{1}=t_{1} /|\Lambda|^{\omega_{1} / 2} \text {. }
$$

The proof of the factorization property (19) at the critical point $\left(\beta_{c}, 0\right)$ is therefore equivalent to showing that the following behaviour occurs $\left(t_{2} \in \mathbb{R}\right)$ :

$$
\begin{gathered}
\lim _{|\Lambda| \rightarrow \infty} U_{4}^{\Lambda}\left(\beta_{c}-t_{2} /|\Lambda|^{\omega_{2} / 2}, 0\right) /|\Lambda|^{2 \omega_{1}}=0, \\
\lim _{|\Lambda| \rightarrow \infty} U_{4}^{\Lambda}\left(\beta_{c}, 0\right) /|\Lambda|^{2 \omega_{1}}=0, \\
\lim _{|\Lambda| \rightarrow \infty}\left\{\left[U_{2}^{\Lambda}\left(\beta_{c}-t_{2} /|\Lambda|^{\omega_{2} / 2}, 0\right)-U_{2}^{\Lambda}\left(\beta_{c}, 0\right)\right] /|\Lambda|^{\omega_{1}}\right\}=0 .
\end{gathered}
$$

The factorization property (19) is thus reduced to the study of the second and 
fourth magnetization cumulants at zero external field. This result is somewhat similar to the now well-known criterion for Gaussianness derived by Newman [16].

Let us also point out that relations (28) clearly indicate how the corresponding laws of large numbers may be recovered within this context: by simply choosing large values for $\omega_{1}$ and $\omega_{2}$. Here, however we are interested by a non-degenerate limiting distribution.

We shall now consider two examples where the limiting bivariate distribution is exactly computable at the critical point.

\section{Example 1: The Curie-Weiss Model (MF)}

This model is defined by the Hamiltonian

$$
H(\{\sigma\})=-\frac{J}{2|\Lambda|}\left(\sum_{i \in \Lambda} \sigma_{i}\right)^{2}-h \sum_{i \in \Lambda} \sigma_{i}
$$

and has been extensively studied by Ellis and Newman [5]. In particular, they have proven that for the classical up and down free spin model:

Lemma 2. At the critical point defined by $\beta=\beta_{c}=1 / J$ and $h=0$, one has:

$$
\lim _{|A| \rightarrow \infty} P\left\{A \leqq \frac{M_{\Lambda}}{|\Lambda|^{3 / 4}}<B\right\}=C_{2} \int_{A}^{B} \exp \left(-C_{1} x^{4}\right) d x,
$$

where $C_{1}$ is a positive constant and $C_{2}$ the normalization factor.

On the other hand, since the energy variable is here given by:

$$
E_{\Lambda}=-\frac{J}{2|\Lambda|}\left(\sum_{i \in \Lambda} \sigma_{i}\right)^{2}=-\frac{J}{2}\left(\frac{M_{\Lambda}}{\sqrt{|\Lambda|}}\right)^{2},
$$

one gets the following

Proposition 3. For the Curie-Weiss model with a classical up and down free spin model, one has at the critical point

$$
\lim _{|\Lambda| \rightarrow \infty} P\left\{a \leqq \frac{E_{\Lambda}-\left\langle E_{\Lambda}\right\rangle}{\sqrt{|\Lambda|}}<b\right\}=\int_{a}^{b} f(y) d y,
$$

where

with

$$
f(y)= \begin{cases}0 & \text { if } y \geqq e \\ C_{2} \frac{1}{\sqrt{-(y-e)}} \exp \left(-C_{1}(y-e)^{2}\right) & \text { if } y<e \\ & \left(C_{1}, C_{2}>0\right)\end{cases}
$$

$$
e=\Gamma(3 / 4) / C_{1}^{1 / 2} \Gamma(1 / 4) .
$$

Proof. Using the results stated in Lemma 2, one easily gets (34-36) by using for instance the convergence of moments (Carleman's condition [17] is here indeed satisfied). 
Using identity (33), it is obvious that the factorization property of Theorem 1 is not allowed here. This can be verified by computing the bivariate moment generating function. As a direct consequence of the preceding result, one gets:

Proposition 4. For the Curie-Weiss model with a classical up and down free spin model, one has at the critical point

$$
\lim _{|\Lambda| \rightarrow \infty}\left\langle\exp \left(t_{1} \tilde{M}_{\Lambda}+t_{2} \tilde{E}_{\Lambda}\right)\right\rangle=\exp \left(t_{2} e\right) C_{2} \int_{-\infty}^{+\infty} \exp \left(t_{1} x-t_{2} J x^{2} / 2\right) \exp \left(-C_{1} x^{4}\right) d x .
$$

This is in complete agreement with the fact that the scaling limit near the critical point (according to $\beta-\tilde{t}_{2}$ ) and at the critical point do not coincide. One indeed has that for

$$
\begin{gathered}
\beta \equiv \beta_{\Lambda}=\beta_{c}-t_{2} /|\Lambda|^{1 / 2}, \\
\lim _{|\Lambda| \rightarrow \infty} P\left\{A \leqq \frac{M_{\Lambda}}{|\Lambda|^{3 / 4}}\left(\beta_{\Lambda}\right)<B\right\}=C_{3} \int_{A}^{B} \exp \left(-C_{1} x^{4}+t_{2} C_{2} x^{2}\right) d x,
\end{gathered}
$$

where $C_{1}$ and $C_{2}$ are real constants and where $C_{3}$ is the normalization factor.

One has so far a good illustration of Theorem 1 . However, due to identity (33), this example may seem somewhat trivial. This is the reason why we shall now consider the edge of a two-dimensional Ising cylinder.

\section{Example 2: The Edge of a Two-Dimensional Ising Cyclinder}

Let us consider the two dimensional Ising cylinder with $2 N$ columns and $2 M$ rows, the Hamiltonian of which may be written as

$$
H(\{\sigma\})=-J \sum_{k=1}^{2 N} \sum_{j=1}^{2 M} \sigma_{j k} \sigma_{j k+1}-J \sum_{j=1}^{2 M-1} \sum_{k=1}^{2 N} \sigma_{j k} \sigma_{j+1 k}
$$

at zero external field with the symmetry of the cylinder (the elementary spin varaibles have been given a double index for convenience). Abraham has already studied the magnetization variable associated to the first row of this model [6]. He proved that, at the critical point defined by $\sinh 2 K_{c}=1$,

$$
\lim _{N \rightarrow \infty} \lim _{M \rightarrow \infty}\left\langle\exp \left(t \sum_{1}^{2 N} \sigma_{1 k} /(N \log N)^{1 / 2}\right)\right\rangle=\exp \left(C t^{2} / 2\right),
$$

where $C>0$ and where the mean value $\langle\cdot\rangle$ is to be taken with respect to (40). Using essentially the same technique, we have computed the following bivariate moment generating function:

$$
\Psi_{2 N}\left(t_{1}, t_{2}\right)=\lim _{M \rightarrow \infty}\left\langle\exp \left(t_{1} \sum_{1}^{2 N} \sigma_{1 k}+t_{2} \sum_{1}^{2 N} \sigma_{1 k} \sigma_{1 k+1}\right)\right\rangle .
$$

We sketch the method for completeness. As in [6], define:

$$
V_{1}(y)=\exp \left(-y^{*} \sum_{1}^{2 N} \sigma_{j}^{z}\right)
$$




$$
\begin{aligned}
\exp \left(-2 y^{*}\right) & =\tanh (y) \\
V_{2}(y) & =\exp \left(y \sum_{1}^{2 N} \sigma_{j}^{x} \sigma_{j+1}^{x}\right),
\end{aligned}
$$

where the $\sigma_{j}^{\alpha}$ denotes the usual Pauli spin operators. By adding a zero row (with coupling $J_{3}$ ) connected with coupling $t_{1}$ to the first row and then letting $J_{3} \rightarrow \infty$, one gets the desired result:

$$
\begin{aligned}
& \Psi_{2 N}\left(t_{1}, t_{2}\right)=\lim _{M \rightarrow \infty} \lim _{K_{3} \rightarrow+\infty}\left[2 \sinh \left(2 t_{1}\right)\right]^{N} \\
& \quad \times\left\{\left\langle 0\left|V_{2}(K)\left[V_{1}(K) V_{2}(K)\right]^{2 M-2} V_{1}(K) V_{2}\left(K-t_{2}\right) V_{1}\left(t_{1}\right) V_{2}\left(K_{3}\right)\right| 0\right\rangle\right\} / \\
& \left\{\langle 0| V_{2}(K)\left[\left(V_{1}(K) V_{2}(K)\right]^{2 M-1}|0\rangle\left\langle 0\left|V_{2}\left(K_{3}\right)\right| 0\right\rangle\right\}\right.
\end{aligned}
$$

with $K=\beta J$. The $|0\rangle$ state corresponds to a state with all spins down: the so-called "vacuum" [18].

Since $V^{\prime}=V_{1}^{1 / 2} V_{2} V_{1}^{1 / 2}$ has a maximal eigenvector for any finite $M$, one gets:

$$
\begin{aligned}
\log \Psi_{2 N}\left(t_{1}, t_{2}\right)= & \sum_{\omega>0}^{<\pi} \log \left\{\cosh ^{2} t_{1} \cdot \cosh \left(2 t_{2} J\right)\right. \\
& +\sinh ^{2} t_{1} \cdot \cosh \left(2 t_{2} J\right) \cdot \operatorname{cotg}(\omega / 2) \cdot \operatorname{tg}\left(\delta^{\prime}(\omega) / 2\right) \cdot \exp \left(2 K^{*}\right) \\
& -\cosh ^{2} t_{1} \cdot \sinh \left(2 t_{2} J\right) \exp \left(-\gamma_{\omega}\right)\left[\exp \left(2 K^{*}\right)\right. \\
& \times(\sinh 2 K-\cosh 2 K \cdot \cos \omega) \\
& \left.+\cosh 2 K \cdot \sin \omega \cdot \operatorname{tg}\left(\delta^{\prime}(\omega) / 2\right)\right]-\sinh ^{2} t_{1} \cdot \sinh \left(2 t_{2} J\right) \\
& \times \operatorname{cotg}(\omega / 2) \cdot \exp \left(-\gamma_{\omega}\right)\left[\exp \left(2 K^{*}\right) \cdot \cosh 2 K \cdot \sin \omega\right. \\
& \left.\left.+\operatorname{tg}\left(\delta^{\prime}(\omega) / 2\right)(\sinh 2 K+\cosh 2 K \cos \omega)\right]\right\} .
\end{aligned}
$$

The values of $\omega$ are given by

$$
\omega=\pi(2 n-1) / 2 N \quad \text { with } n=1,2, \ldots, N,
$$

and $\delta^{\prime}(\omega)$ is given as usual by

$$
\begin{aligned}
\operatorname{tg}\left(\delta^{\prime}(\omega) / 2\right)= & \sinh 2 K \cdot \sin \omega /\left(\sinh \gamma_{\omega}+\sinh 2 K^{*}\right. \\
& \left.\times \cosh 2 K-\cosh 2 K^{*} \sinh 2 K \cos \omega\right) .
\end{aligned}
$$

$\gamma_{\omega}$ is Onsager's function, i.e. the positive solution of

$$
\cosh \gamma_{\omega}=\cosh 2 K \cdot \operatorname{coth} 2 K-\cos \omega .
$$

The marginal cumulant generating functions can directly be derived from (43). One obtains

$$
\begin{aligned}
\log \Psi_{2 N}\left(t_{1}, 0\right)= & \sum_{\omega>0}^{<\pi} \log \left\{\cosh ^{2} t_{1}+\sinh ^{2} t_{1} \operatorname{cotg}(\omega / 2) .\right. \\
& \left.\times \operatorname{tg}\left(\delta^{\prime}(\omega / 2)\right) \exp \left(2 K^{*}\right)\right\}
\end{aligned}
$$


for the block magnetization variable and

$$
\begin{aligned}
\log \Psi_{2 N}\left(0, t_{2}\right)= & \sum_{\omega>0}^{<\pi} \log \left\{\cosh \left(2 t_{2} J\right)-\sinh \left(2 t_{2} J\right) \exp \left(-\gamma_{\omega}\right)\right. \\
& \times\left[\exp \left(2 K^{*}\right)(\sinh 2 K-\cosh 2 K \cos \omega)\right. \\
& \left.\left.+\cosh 2 K \cdot \sin \omega \cdot \operatorname{tg}\left(\delta^{\prime}(\omega) / 2\right)\right]\right\}
\end{aligned}
$$

for the block energy variable. The fluctuations of the first row magnetization variable at zero external field are thus (in agreement with [6]):

$$
\begin{aligned}
& \lim _{M \rightarrow \infty}\left\langle\sum_{1}^{2 N} \sigma_{1 k} ; \sum_{1}^{2 N} \sigma_{1 k}\right\rangle \\
& =2 \sum_{\omega>0}^{<\pi}\left\{1+\exp \left(2 K^{*}\right) \operatorname{tg}\left(\delta^{\prime}(\omega) / 2\right) \operatorname{cotg}(\omega / 2)\right\},
\end{aligned}
$$

and those of the first row energy variable are given by

$$
\begin{aligned}
& \lim _{M \rightarrow \infty}\left\langle-J \sum_{1}^{2 N} \sigma_{1 k} \sigma_{1 k+1} ;-J \sum_{1}^{2 N} \sigma_{1 k} \sigma_{1 k+1}\right\rangle \\
& =4 J^{2} \sum_{\omega>0}^{<\pi}\left\{1-\left(\operatorname{coth} 2 K-\exp \left(2 K^{*}-\gamma_{\omega}\right) / \sinh 2 K\right)^{2}\right\} .
\end{aligned}
$$

We are now able to give the following

Lemma 5. At the critical point given by $\sinh 2 K_{c}=1$, one gets

$$
\begin{gathered}
\lim _{M, N \rightarrow \infty} \frac{1}{N \log N}\left\langle\sum_{1}^{2 N} \sigma_{1 k} ; \sum_{1}^{2 N} \sigma_{1 k}\right\rangle<+\infty \\
\lim _{M, N \rightarrow \infty} \frac{1}{N}\left\langle-J \sum_{1}^{2 N} \sigma_{1 k} \sigma_{1 k+1} ; \quad-J \sum_{1}^{2 N} \sigma_{1 k} \sigma_{1 k+1}\right\rangle<+\infty
\end{gathered}
$$

Proof. Property (53) has already been proved in $[6,12]$ and relation (54) follows easily from (52)

These results suggest of course the following renormalization procedure for the (magnetization, energy) bivariate variable at the critical point:

$$
\Phi\left(t_{1}, t_{2}\right)=\lim _{N \rightarrow \infty}\left\langle\exp \left(t_{1} M_{2 N} /(N \log N)^{1 / 2}+t_{2} \bar{E}_{2 N} / N^{1 / 2}\right)\right\rangle
$$

for the zero mean valued variables

$$
\begin{aligned}
M_{2 N} & =\sum_{1}^{2 N} \sigma_{1 k}, \\
\bar{E}_{2 N} & =-J \sum_{1}^{2 N} \sigma_{1 k} \sigma_{1 k+1}+\left\langle J \sum_{1}^{2 N} \sigma_{1 k} \sigma_{1 k+1}\right\rangle .
\end{aligned}
$$

We are thus led to the following

Theorem 6. At the critical point and with the previously defined notations; one has for 
any real $t_{1}, t_{2}$ :

$$
\lim _{M, N \rightarrow \infty}\left\langle\exp \left(t_{1} M_{2 N} /(N \log N)^{1 / 2}+t_{2} \bar{E}_{2 N} / N^{1 / 2}\right)\right\rangle=\exp \left(C_{1} t_{1}^{2}\right) \exp \left(C_{2} t_{2}^{2}\right),
$$

where $C_{1}$ and $C_{2}$ are positive real constants.

Proof. As explained in Sect. 2, one may easily show that, at the critical point,

$$
\Psi_{2 N}\left(t_{1}, t_{2}\right)=\lim _{M \rightarrow \infty} \frac{\left\langle\exp \left(t_{1} M_{2 N}+t_{2} \widetilde{E}_{2 N}\right)\right\rangle}{\left\langle\exp \left(t_{1} M_{2 N}\right)\right\rangle\left\langle\exp \left(t_{2} \bar{E}_{2 N}\right)\right\rangle}=\lim _{M \rightarrow \infty} \frac{\left\langle\exp \left(t_{1} M_{2 N}\right)\right\rangle^{\prime}}{\left\langle\exp \left(t_{1} M_{2 N}\right)\right\rangle}
$$

with $\langle\cdot\rangle^{\prime}$ denoting the expectation value with respect to $(40)$ where the contribution of the first row $\beta_{c} J \sum_{1}^{2 N} \sigma_{1 k} \sigma_{1 k+1}$ has to be modified by $\left(\beta_{c}-t_{2}\right) \sum_{1}^{2 N} \sigma_{1 k} \sigma_{1 k+1}$.

From (45) one obtains

$$
\Psi_{2 N}\left(t_{1}, t_{2}\right)=\prod_{\omega} \frac{\cosh ^{2} t_{1}+\sinh ^{2} t_{1} \operatorname{cotg}(\omega / 2) F_{\omega}\left(\widehat{K}, K^{*}\right)}{\cosh ^{2} t_{1}+\sinh ^{2} t_{1} \operatorname{cotg}(\omega / 2) F_{\omega}\left(K, K^{*}\right)},
$$

where

$$
F_{\omega}\left(\hat{K}, K^{*}\right)=\frac{(\cosh 2 \hat{K}+\sinh 2 \hat{K} \cos \omega) \operatorname{tg}\left(\delta^{\prime}(\omega) / 2\right)+\sinh 2 \hat{K} \sin \omega \exp \left(2 K^{*}\right)}{\sinh 2 \hat{K} \sin \omega \operatorname{tg}\left(\delta^{\prime}(\omega) / 2\right)+(\cosh 2 \hat{K}-\sinh 2 \widehat{K} \cos \omega) \exp \left(2 K^{*}\right)}
$$

with

$$
\hat{K}=K-t_{2} J \text {. }
$$

We first would like to prove the factorization property of the limiting bivariate moment generating function. According to (30), one therefore has to study the second and fourth derivatives of the logarithm of $\Psi_{2 N}\left(t_{1}, t_{2}\right)$ at $t_{1}=0$.

Let

$$
\begin{array}{r}
U_{2}(\beta, 0)=\left.\partial_{t_{1}}^{2} \lim _{M \rightarrow \infty} \log \left\langle\exp \left(t_{1} M_{2 N}\right)\right\rangle\right|_{\beta, 0}, \\
U_{2}^{\prime}\left(\beta-t_{2}, 0\right)=\left.\partial_{t_{1}}^{2} \lim _{M \rightarrow \infty} \log \left\langle\exp \left(t_{1} M_{2 N}\right)\right\rangle^{\prime}\right|_{\beta, 0},
\end{array}
$$

then

$$
\begin{array}{r}
U_{2}(\beta, 0)=2 \sum_{\omega>0}^{<\pi}\left(1+\operatorname{cotg}(\omega / 2) \cdot F_{\omega}\left(K, K^{*}\right)\right), \\
U_{2}^{\prime}\left(\beta-t_{2}, 0\right)=2 \sum_{\omega>0}^{<\pi}\left(1+\operatorname{cotg}(\omega / 2) F_{\omega}\left(\hat{K}, K^{*}\right)\right) .
\end{array}
$$

In the same way, one obtains

$$
\begin{gathered}
U_{4}(\beta, 0)=-4 \sum_{\omega>0}^{<\pi}\left\{1+4 \operatorname{cotg}(\omega / 2) F_{\omega}\left(K, K^{*}\right)+3 \operatorname{cotg}^{2}(\omega / 2) \cdot F_{\omega}^{2}\left(K, K^{*}\right)\right\} \\
U_{4}^{\prime}\left(\beta-t_{2}, 0\right)=-4 \sum_{\omega>0}^{<\pi}\left\{1+4 \operatorname{cotg}(\omega / 2) F_{\omega}\left(\hat{K}, K^{*}\right)+3 \operatorname{cotg}^{2}(\omega / 2) F_{\omega}^{2}\left(\hat{R}, K^{*}\right)\right\} .
\end{gathered}
$$


In appendices 1, 2 and 3 we prove respectively that

$$
\begin{array}{r}
\lim _{N \rightarrow \infty} U_{4}\left(\beta_{c}, 0\right) / N^{2} \log ^{2} N=0, \\
\lim _{N \rightarrow \infty} U_{4}^{\prime}\left(\beta_{c}-t_{2} / \sqrt{N}, 0\right) / N^{2} \log ^{2} N=0, \\
\lim _{N \rightarrow \infty} \frac{U_{2}^{\prime}\left(\beta_{c}-t_{2} / \sqrt{N}, 0\right)-U_{2}\left(\beta_{c}, 0\right)}{N \log N}=0 .
\end{array}
$$

According to (30), this implies the validity of the factorization property at the critical point:

$$
\lim _{M, N \rightarrow \infty}\left\langle\exp \left(t_{1} \tilde{M}_{2 N}+t_{2} \widetilde{E}_{2 N}\right)\right\rangle=E\left\{\exp \left(t_{1} X\right)\right\} E\left\{\exp \left(t_{2} Y\right)\right\},
$$

where $X$ and $Y$ are defined by

$$
\begin{aligned}
\tilde{M}_{2 N} & =\frac{M_{2 N}}{(N \log N)^{1 / 2}} \underset{M, N \rightarrow \infty}{\longrightarrow} X, \\
\tilde{E}_{2 N} & =\frac{E_{2 N}-\left\langle E_{2 N}\right\rangle}{\sqrt{N}} \underset{M, N \rightarrow \infty}{\longrightarrow} Y .
\end{aligned}
$$

We already know that $X$ has a Gaussian distribution [6]. To achieve the proof of Theorem 6, it remains to show that the probability distribution of $Y$ is indeed Gaussian. This may easily be done starting from (48) and using standard techniques of probability theory.

Comment. ${ }^{1}$ This result may seem in conflict with the divergence of the boundary specific heat at zero external field as obtained by McCoy and $\mathrm{Wu}$ [19] and Fisher and Ferdinand [20]. It should however be stressed that the reference system they use is not identical with ours. They are indeed interested by the function

$$
Z_{\text {cylinder }}(x, y) / Z_{\text {torus }}(x, 0)
$$

with a field " $y$ " only applied to the edges of the cylinder. This allows ${ }^{1}$ to define the corresponding boundary free energy and, by considering the second tempeature derivative, to obtain the boundary specific heat which behaves as $\left(T-T_{c}\right)^{-1}$.

In our probabilistic approach however, we are interested by a moment generating function, i.e.

$$
Z_{\text {cylinder }}\left(x-t \cdot \delta_{\text {first row }}, y\right) / Z_{\text {cylinder }}(x, 0),
$$

where $t$ modifies the temperature of the first row only, the second temperature derivative with respect to $t$ of which leads to the fluctuation of the first row energy:

$$
\left\langle-\sum_{1}^{2 N} \sigma_{1 k} \sigma_{1 k+1} ; \quad-J \sum_{1}^{2 N} \sigma_{1 k} \sigma_{1 k+1}\right\rangle,
$$

which in this case is not identical to the boundary specific heat. It should be pointed

1 The author wishes to thank M. E. Fisher for a clarifying correspondence about this point 
out that no divergence occurs in (77); this guarantees the Gaussianness of the $Y$ variable.

\section{Concluding Remarks}

In this paper on limiting bivariate (magnetization, energy) probability distributions, we have established a simple criterion (30) to study the independence of the limiting variables associated to the magnetization $M_{\Lambda}$ and the energy $E_{\Lambda}$.

We have seen how a "strong" dependence between $M_{\Lambda}$ and $E_{\Lambda}$ (i.e. such that they become non-independent) is connected to critical point exponent equalities (cf. Sect. 1) and how a "weak" dependence (i.e. such that they become independent) leads to a unique scaling limit (cf. (20)) or, equivalently, to a unique fixed point within the renormalization group formalism.

Our study of the Curie-Weiss model along this line shows once more the peculiarness of this model. In particular we have proven how the strong dependence between $M_{A}$ and $E_{A}$ (cf. (33)) in this model leads to a non-unique scaling limit.

The edge of a two-dimensional Ising cylinder gives us a second example where the previous analysis may be performed exactly. In fact, we have shown that this model is characterized by a weak dependence (in the previously defined sense) between $M_{\Lambda}$ and $E_{\Lambda}$ (cf. (58)). It should however be stressed that edge properties like this one may well turn out to be completely different from the corresponding bulk ones. It would therefore be interesting to extend this study to $d$-dimensional Ising ferromagnets.

Finally, let us point out that it would be extremely interesting, in particular for the $d=3$ Ising ferromagnet, to study the relation which may exist between unicity and triviality of the scaling limit.

\section{Appendix 1}

Let us prove that, at the critical point,

$$
\lim _{N \rightarrow \infty} U_{4}\left(\beta_{c}, 0\right) / N^{2} \log ^{2} N=0 .
$$

This is equivalent to show that

$$
\begin{aligned}
& \lim _{N \rightarrow \infty} \frac{1}{N^{2} \log ^{2} N} \sum_{\omega>0}^{<\pi}\left\{1+4 \operatorname{cotg}(\omega / 2) \operatorname{tg}\left(\frac{\delta^{\prime}(\omega)}{2}\right)\right. \\
& \left.\times \exp \left(2 K_{c}^{*}\right)+3 \operatorname{cotg}^{2}(\omega / 2) \operatorname{tg}^{2}\left(\frac{\delta^{\prime}(\omega)}{2}\right) \exp \left(4 K_{c}^{*}\right)\right\}=0
\end{aligned}
$$

where $K_{c}$ is defined by

Since

$$
\sinh 2 K_{c}=1
$$

$$
\operatorname{cotg}(\omega / 2) \operatorname{tg}\left(\frac{\delta^{\prime}(\omega)}{2}\right)=\frac{2 \sinh 2 K \cos ^{2}(\omega / 2)}{\sinh \gamma_{\omega}+\sinh 2 K^{*} \cosh 2 K-\cosh 2 K^{*} \sinh 2 K \cos \omega},
$$


one easily gets at $K=K_{c}$,

$$
\left.\operatorname{cotg}(\omega / 2) \operatorname{tg}\left(\frac{\delta^{\prime}(\omega)}{2}\right)\right|_{K_{c}} \leqq \frac{1}{\sin (\omega / 2)}
$$

It remains to use the following inequality $(0<\omega<\pi)$ [12]

$$
\left|\sin ^{-1}(\omega / 2)-(\omega / 2)^{-1}\right|<C^{t}
$$

to show that

1) $\sum_{\omega>0}^{<\pi} \operatorname{cotg}(\omega / 2) \operatorname{tg}\left(\frac{\delta^{\prime}(\omega)}{2}\right)$ does not grow faster than $N \log N$, since indeed $\sum_{\omega>0}^{<\pi}(1 / \omega)$ grows like $N \log N$.

2) $\sum_{\omega>0}^{<\pi}\left[\operatorname{cotg}(\omega / 2) \operatorname{tg}\left(\frac{\delta^{\prime}(\omega)}{2}\right)\right]^{2}$ does not grow faster than $N^{2}$ for large enough $N$ since indeed $\sum_{\omega>0}^{<\pi}\left(1 / \omega^{2}\right)$ grows like $N^{2}$.

This achieves the proof of (A.1)

\section{Appendix 2}

Let us prove that

$$
\lim _{N \rightarrow \infty} U_{4}^{\prime}\left(\beta_{c}-t_{2} / \sqrt{N}, 0\right) / N^{2} \log ^{2} N=0
$$

where

$$
\begin{aligned}
U_{4}^{\prime}\left(\beta-t_{2}, 0\right)= & -4 \sum_{\omega>0}^{<\pi}\left\{1+4 \operatorname{cotg}(\omega / 2) F_{\omega}\left(\hat{K}, K^{*}\right)\right. \\
& \left.+3 \operatorname{cotg}^{2}(\omega / 2) F_{\omega}^{2}\left(\hat{K}, K^{*}\right)\right\}
\end{aligned}
$$

with

$$
\begin{gathered}
\hat{K}=\left(\beta-t_{2}\right) J \\
F_{\omega}\left(\hat{K}, K^{*}\right)=\frac{(\cosh 2 \hat{K}+\sinh 2 \hat{K} \cos \omega) \operatorname{tg}\left(\delta^{\prime}(\omega) / 2\right)+\sinh 2 \hat{K} \sin \omega \exp \left(2 K^{*}\right)}{\sinh 2 \hat{K} \sin \omega \operatorname{tg}\left(\delta^{\prime}(\omega) / 2\right)+(\cosh 2 \hat{K}-\sinh 2 \hat{K} \cos \omega) \exp \left(2 K^{*}\right)} .
\end{gathered}
$$

Now, by continuity with respect to $\hat{K}$, one may prove that

$$
\begin{aligned}
\forall \varepsilon> & 0 \exists N_{0}>0: N>N_{0} \Rightarrow \mid \operatorname{cotg}(\omega / 2) F_{\omega}\left(\tilde{K}_{c}, K_{c}^{*}\right) \\
& -\operatorname{cotg}(\omega / 2) F_{\omega}\left(K_{c}, K_{c}^{*}\right) \mid \leqq \varepsilon / \sin (\omega / 2)
\end{aligned}
$$

with $0<\omega \leqq \pi$ and

$$
\widetilde{K}_{c}=K_{c}-t_{2} J / \sqrt{N}
$$


Therefore

$$
\left|\sum_{\omega>0}^{<\pi} \operatorname{cotg}(\omega / 2) F_{\omega}\left(\tilde{K}_{c}, K_{c}^{*}\right)\right| \leqq \sum_{\omega>0}^{<\pi} \operatorname{cotg}(\omega / 2) F_{\omega}\left(K_{c}, K_{c}^{*}\right)+2 \varepsilon \sum_{\omega>0}^{<\pi} \frac{1}{\omega}+\varepsilon \cdot C^{t}
$$

and, thus, the left-hand side of this inequality does not grow faster than $N \log N$. It is also straightforward to prove that

$$
\begin{aligned}
\forall \varepsilon> & 0 \exists N_{0}>0: N>N_{0} \Rightarrow \mid \operatorname{cotg}^{2}(\omega / 2) F_{\omega}^{2}\left(\tilde{K}_{c}, K_{c}^{*}\right) \\
& -\operatorname{cotg}^{2}(\omega / 2) F_{\omega}^{2}\left(K_{c}, K_{c}^{*}\right) \mid \leqq \frac{\varepsilon}{\sin ^{2}(\omega / 2)} .
\end{aligned}
$$

For large enough $N$, this leads to

$$
\left|\sum_{\omega>0}^{<\pi} \operatorname{cotg}^{2}(\omega / 2) F_{\omega}^{2}\left(\tilde{K}_{c}, K_{c}^{*}\right)\right| \leqq C^{t} \cdot N^{2},
$$

which achieves the proof of (A.7).

\section{Appendix 3}

Let us prove that

$$
\lim _{N \rightarrow \infty} \frac{U_{2}^{\prime}\left(\beta_{c}-t_{2} / \sqrt{N}, 0\right)-U_{2}\left(\beta_{c}, 0\right)}{N \log N}=0
$$

where

$$
\begin{aligned}
& U_{2}^{\prime}\left(\beta_{c}-t_{2} / \sqrt{N}, 0\right)-U_{2}(\beta, 0) \\
& \quad=\sum_{\omega>0}^{<\pi} \operatorname{cotg}(\omega / 2)\left\{F_{\omega}\left(K_{c}-t_{2} J / \sqrt{N}, K_{c}^{*}\right)-F_{\omega}\left(K_{c}, K_{c}^{*}\right)\right\} .
\end{aligned}
$$

Using (A.11), one gets

$$
\frac{\left|U_{2}^{\prime}\left(\beta_{c}-t_{2} / \sqrt{N}, 0\right)-U_{2}\left(\beta_{c}, 0\right)\right|}{N \log N} \leqq \varepsilon \frac{1}{N \log N} \sum_{\omega>0}^{<\pi} 1 / \sin (\omega / 2)
$$

It remains to use (A.6) to obtain

$$
\frac{\left|U_{2}^{\prime}\left(\beta_{c}-t_{2} / \sqrt{N}, 0\right)-U_{2}\left(\beta_{c}, 0\right)\right|}{N \log N} \leqq \varepsilon C^{t}
$$

for large enough $N$. Since $\varepsilon$ is arbitrarily small, one easily obtains (A.16).

Acknowledgements. The author is grateful to D. B. Abraham, D. C. Brydges, Ph. de Gottal, F. Dunlop, C. M. Newman, B. Simon and A. Wightman for stimulating discussions. He also wishes to thank M. E.

Fisher and J. Fröhlich for useful correspondence. 


\section{References}

1. Jona-Lasinio, G.: The renormalization group: A probabilistic view. Nuovo Cim. 26B, 99-119 (1975)

2. Cassandro, M. Jona-Lasinio, G.: Critical point behaviour and probability theory. Adv. Phys. 27, 913-941 (1978)

3. Sinaï, Y. G.: Mathematical foundations of the renormalization group method. In: Statistical physics and mathematical problems in theoretical physics. Dell' Antonio, G., Doplicher, S., Jona-Lasinio, G. (eds.). Lectures Notes in Physics, Vol. 80, Berlin, Heidelberg, New York: Springer 1978

4. Newman, C. M.: Critical point inequalities and scaling limits. Commun. Math. Phys. 66, 181-196 (1979)

5. Ellis, R. S., Newman, C. M.: The statistics of Curie-Weiss models. J. Stat. Phys. 19, 149-161 (1978)

6. Abraham, D. B.: Block spins in the edge of an Ising ferromagnetic half-plane. J. Stat. Phys. 19, 553556 (1978)

7. Aizenman, M.: Geometric analysis of $\phi^{4}$ fields and Ising models. Parts I and II. Commun. Math. Phys. 86, 1-48 (1982)

8. Fröhlich, J.: On the triviality of $\lambda \phi_{d}^{4}$ theories and the approach to the critical point in $d \gtrsim 4$ dimensions. Nucl. Phys. B200, [FS4] 281-296 (1982)

9. Aizenman, M., Graham, R.: On the renormalized coupling constant and the susceptibility in $\phi_{4}^{4}$ field theory and the Ising model in four dimensions. Nucl. Phys. B225, [FS9] 261-288 (1983)

10. Newman, C. M.: Normal fluctuations and the FKG inequalities. Commun. Math. Phys. 74, 119-128 (1980)

11. Iagolnitzer, D., Souillard, B.: Lee-Yang theory and normal fluctuations. Phys. Rev. B19, 1515-1518 (1979)

12. De Coninck, J.: Scaling limit of the energy variable for the two-dimensional Ising ferromagnet. Commun. Math. Phys. 95, 53-59 (1984)

13. Newman, C. M.: A general central limit theorem for FKG systems. Commun. Math. Phys. 91, 75-80 (1983)

14. Lee, T. D., Yang, C. N.: Statistical theory of equations of states and phase transitions. II. Lattice gas and Ising model. Phys. Rev. 87, 410-419 (1952)

15. De Coninck, J., de Gottal, Ph.: New inequalities for Ising ferromagnets, J. Stat. Phys. 36, 181-188 (1984)

16. Newman, C. M.: Inequalities for Ising model and field theories which obey the Lee-Yang theorem. Commun. Math. Phys. 41, 1-9 (1975)

17. Breiman, L.: Probability. Reading, MA: Addison-Wesley 1968

18. Schultz, T. D., Mattis, D. C., Lieb, E. H.: The two-dimensional Ising model as a soluble problem of many fermions. Rev. Mod. Phys. 36, 856-871 (1964)

19. McCoy, B. M., Wu, T.-T.: The two-dimensional Ising model. Cambridge Massachussetts: Harward University Press 1973

20. Fisher, M. E., Ferdinand, A. E.: Interfacial boundary, and size effects at critical points. Phys. Rev. Lett. 19, 169-172 (1967)

21. Hecht, R.: Correlation functions for the two-dimensional Ising model. Phys. Rev. 158, 557-561 (1967)

22. Aizenman, M.: Rigorous results on the critical behavior in statistical mechanics. In: Scaling and selfsimilarity in physics: Renormalization in statistical mechanics and dynamics. Fröhlich, J., (ed.). Boston: Birkhauser 1983

23. Fröhlich, J., Spencer, T.: Some recent rigorous results in the theory of phase transitions and critical pehnomena. Séminaire Bourbaki, $n^{\circ} 586(1981 / 82)$

\section{Communicated by J. Fröhlich}

Received March 1, 1986; in revised form September 24, 1986 
